\title{
Retraction
}

\section{Retracted: Performance of Water-Based Liquid Scintillator: An Independent Analysis}

\section{Advances in High Energy Physics}

Received 5 January 2015; Accepted 5 January 2015

Copyright (C) 2015 Advances in High Energy Physics. This is an open access article distributed under the Creative Commons Attribution License, which permits unrestricted use, distribution, and reproduction in any medium, provided the original work is properly cited. The publication of this article was funded by SCOAP ${ }^{3}$.

The paper titled "Performance of Water-Based Liquid Scintillator: An Independent Analysis" [1], published in Advances in High Energy Physics has been retracted by the authors as they did not have full permission to use the data to submit the manuscript.

\section{References}

[1] D. Beznosko, A. Batyrkhanov, A. Duspayev, A. Iakovlev, and M. Yessenov, "Performance of water-based liquid scintillator: an independent analysis," Advances in High Energy Physics, vol. 2014, Article ID 250646, 8 pages, 2014. 

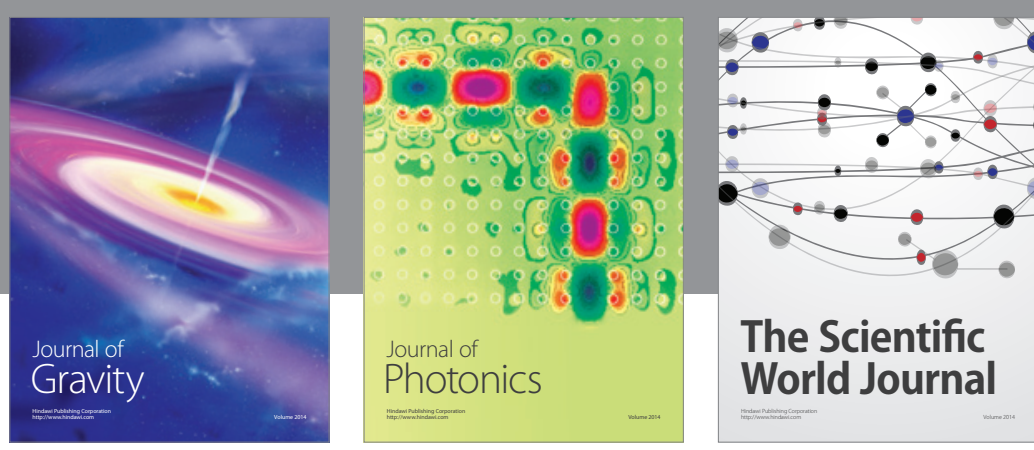

The Scientific World Journal
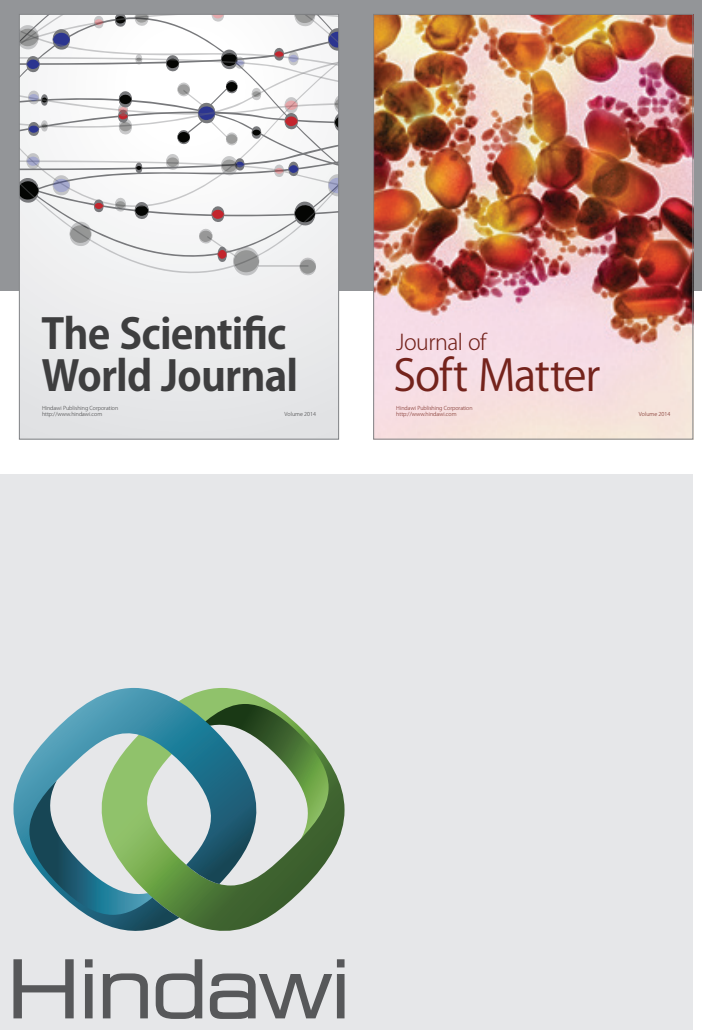

Submit your manuscripts at

http://www.hindawi.com

nternational Journal of

Statistical Mechanics
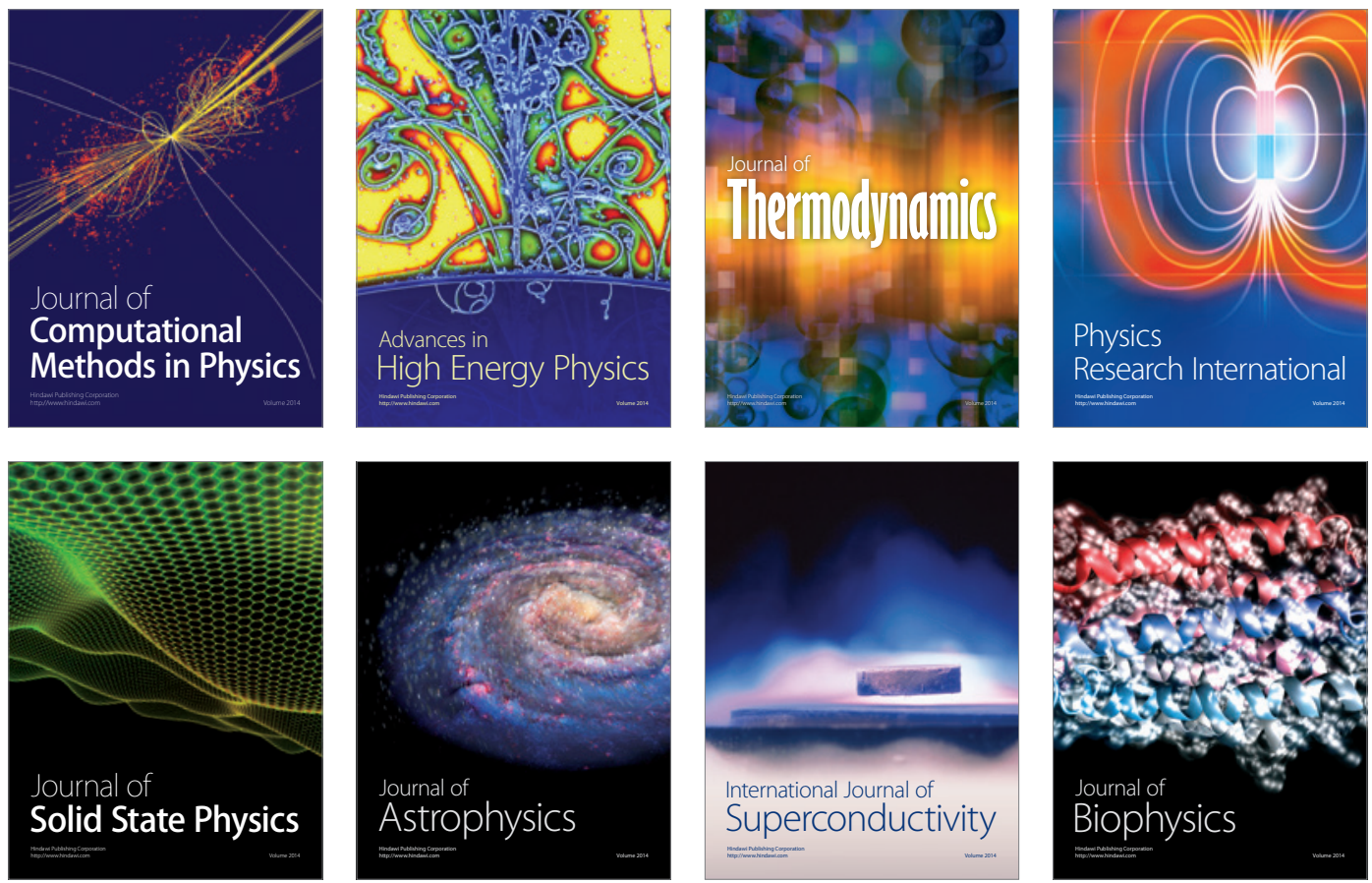
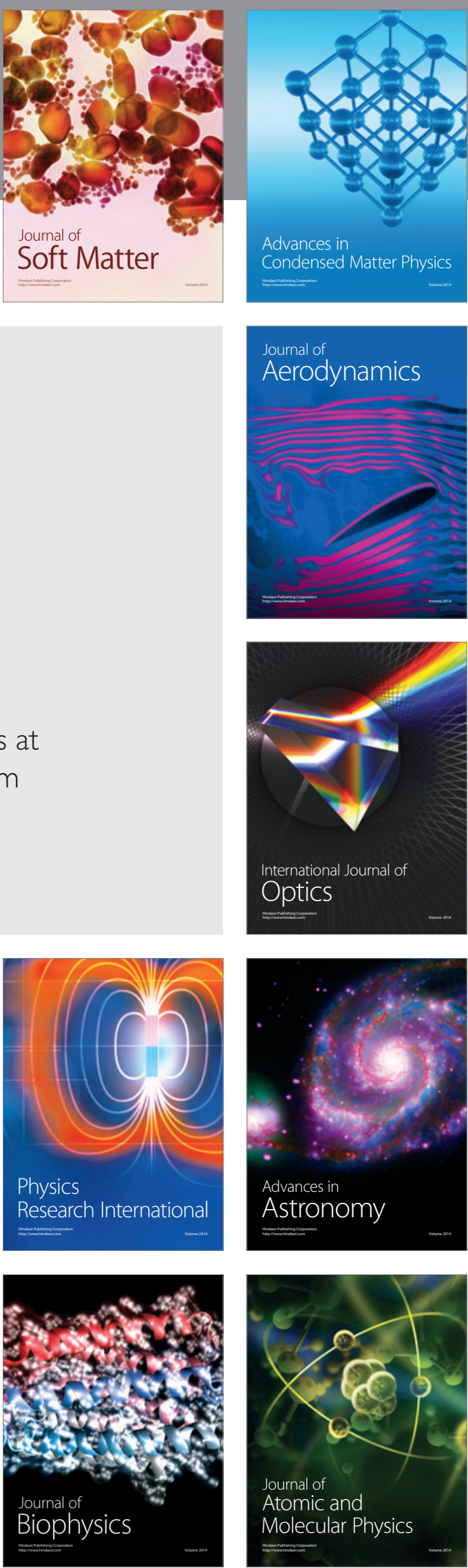